\title{
Extragalactic large-scale structures behind the southern Milky Way ${ }^{\star}$
}

\section{Redshifts obtained with MEFOS}

\author{
P. A. Woudt ${ }^{1}$, R. C. Kraan-Korteweg ${ }^{2}$, V. Cayatte ${ }^{3}$, C. Balkowski ${ }^{3}$, and P. Felenbok ${ }^{4}$
}

1 Department of Astronomy, University of Cape Town, Rondebosch 7700, South Africa

2 Depto. de Astronomía, Universidad de Guanajuato, Apartado Postal 144, Guanajuato, Gto 36000, Mexico

3 Obs. de Paris, GEPI, CNRS and Université Paris 7, 5 place Jules Janssen, 92195 Meudon Cedex, France

4 Obs. de Paris, LUTH, CNRS and Université Paris 7, 5 place Jules Janssen, 92195 Meudon Cedex, France

Received 24 June 2003 / Accepted 7 November 2003

\begin{abstract}
As part of our efforts to unveil extragalactic large-scale structures behind the southern Milky Way, we here present redshifts for 764 galaxies in the Hydra/Antlia, Crux and Great Attractor region $\left(266^{\circ} \leq \ell \leq 338^{\circ},|b| \lesssim 10^{\circ}\right)$, obtained with the Meudon-ESO Fibre Object Spectrograph (MEFOS) at the 3.6-m telescope of ESO. The observations are part of a redshift survey of partially obscured galaxies recorded in the course of a deep optical galaxy search behind the southern Milky Way (Kraan-Korteweg 2000; Woudt \& Kraan-Korteweg 2001). A total of 947 galaxies have been observed, a small percentage of the spectra $(N=109,11.5 \%)$ were contaminated by foreground stars, and 74 galaxies $(7.8 \%)$ were too faint to allow a reliable redshift determination. With MEFOS we obtained spectra down to the faintest galaxies of our optical galaxy survey, and hence probe large-scale structures out to larger distances $\left(v \lessgtr 30000 \mathrm{~km} \mathrm{~s}^{-1}\right)$ than our other redshift follow-ups using the 1.9-m telescope at the South African Astronomical Observatory (Kraan-Korteweg et al. 1995; Fairall et al. 1998; Woudt et al. 1999) and the 64-m Parkes radio telescope (Kraan-Korteweg et al. 2002). The most distinct large-scale structures revealed in the southern Zone of Avoidance are discussed in context to known structures adjacent to the Milky Way.
\end{abstract}

Key words. catalogs - surveys - ISM: dust, extinction - galaxies: distances and redshifts - clusters: individual: ACO 3627 large-scale structure of Universe

\section{Introduction}

At the same time when significant advances are made in characterising large-scale structures of galaxies in the Universe through dedicated surveys such as the Sloan Digital Sky Survey (Zehavi et al. 2002) and the 2dF Galaxy Redshift Survey (Colless et al. 2001), the study of large-scale structures behind the Milky Way has also progressed enormously. Dedicated deep optical searches, near infrared all-sky surveys (2MASS and DENIS), HI all-sky surveys and X-ray surveys, have all resulted in the detection of voids, clusters and superclusters at low Galactic latitude (for a review, see Kraan-Korteweg \& Lahav (2000) and references therein).

We have focussed our efforts on an extended region behind the southern Milky Way (Kraan-Korteweg 2000;

Send offprint requests to: $\mathrm{P}$. A. Woudt, e-mail: pwoudt@circinus.ast.uct.ac.za

* Tables 1 and 2 are only available in electronic form at the CDS via anonymous ftp to cdsarc.u-strasbg.fr $(130.79 .128 .5)$ or via http://cdsweb.u-strasbg.fr/cgi-bin/qcat?J/A+A/415/9 Based on observations taken at the European Southern Observatory, La Silla, Chile.
Woudt \& Kraan-Korteweg 2001). This region of space harbours a large local overdensity of galaxies, the Great Attractor (Dressler et al. 1987; Lynden-Bell et al. 1988; Kolatt et al. 1995; Tonry et al. 2000). Local and complex large-scale structures appear to extend across the Galactic Plane (GP) in this part of the sky (Kraan-Korteweg et al. 1995 (Paper I); Fairall et al. 1998 (Paper II); Woudt et al. 1999 (Paper III)).

Our deep optical galaxy search behind the southern Milky Way is divided into three separate areas: the Hydra/Antlia region $\left(266^{\circ} \leq \ell \leq 296^{\circ},-10^{\circ} \leq b \leq+8^{\circ}\right.$ ) (Kraan-Korteweg 2000), the Crux region $\left(289^{\circ} \leq \ell \leq 318^{\circ},|b| \leq 10^{\circ}\right)$, and the Great Attractor region $\left(316^{\circ} \leq \ell \leq 338^{\circ},|b| \leq 10^{\circ}\right.$ ) (Woudt \& Kraan-Korteweg 2001). The optical survey in these regions has revealed over 11000 previously unrecorded galaxies with observed major diameters $\gtrsim 0$ '.2. Recently, the survey has been extended towards the Puppis region (at lower Galactic longitudes), and the Scorpius region (towards the Galactic bulge).

Following our deep optical galaxy search, we have been engaged in a redshift survey of these newly found partially obscured galaxies. This redshift survey consists of three 
different, yet complementary approaches in tracing the extragalactic large-scale structures. They are:

- Optical spectroscopy of individual early-type galaxies with the 1.9-m telescope of the South African Astronomical Observatory (SAAO) (Papers I-III).

- HI line spectroscopy of low surface brightness spiral and irregular galaxies with the Parkes 64-m radio telescope (Kraan-Korteweg et al. 2002).

- Low resolution, multi-fibre spectroscopy of galaxies in regions of high galaxy-density with the 3.6-m telescope of the European Southern Observatory (ESO), initially in combination with Optopus, later with MEFOS (Meudon-ESO Fibre Object Spectrograph).

MEFOS, with its 29 object fibres and large field of view (1-degree diameter), is optimal for covering the highest density regions in our optical survey. These multi-fibre observations are an important part of our redshift survey, specifically in mapping the centre of the GA region and the extended overdensity around ACO 3627.

In this paper - Paper IV in the series of papers on extragalactic large-scale structures behind the southern Milky Way - the results from the MEFOS multi-fibre spectroscopy in the Hydra/Antlia, Crux and Great Attractor regions are presented.

\section{Observations and data reduction}

MEFOS mounted at the prime focus of the ESO 3.6-m telescope, provided a large field of view (1 degree in diameter). It had 30 arms that could point within the $20 \mathrm{~cm}$ (1 degree) field. One arm was used for guiding, while the other 29 arms were dedicated to the astronomical objects. Each arm was equipped with an individual viewing system for accurate setting - very useful for objects in star crowded regions - and carried two spectroscopic fibres, one for the astronomical object, and the other one for the sky recording needed for sky subtraction. The spectral fibres intercepted 2.5 arcsec on the sky.

The data presented in this paper were obtained during 10.5 nights in four observing runs between February 1993 and May 1995. A total of 48 fields were observed in our surveyed region, i.e., the Hydra/Antlia, Crux and Great Attractor (GA) regions. One field was observed twice in order to check the stability and performance of MEFOS. Also, by observing one field twice, the uncertainties in the redshifts obtained following the cross-correlation procedure (Tonry \& Davies 1979) can be quantified (see Sect. 2.2).

In all the observing runs, the CCD Tek \#32 was used in combination with grating \#15. The wavelength coverage spans a range of $3815 \AA-6120 \AA$, with a dispersion of $170 \AA / \mathrm{mm}$ and a resolution of $\sim 11 \AA$.

Before and after each science frame, an arc exposure was made. In our case, the arcs were made with a helium-neon lamp. A filter (BG 28) was put in front of the lamp to reduce saturation of the bright lines at the red end of the spectrum. The wavelength-calibration frames had exposure times between one and two minutes, the science frames had exposure times of 20 or $30 \mathrm{~min}$. Generally, each MEFOS field has been observed for 1 hour in total (combining 2 or 3 individual exposures).
The upper panel of Fig. 1 shows the distribution of the observed MEFOS fields in the Hydra/Antlia, Crux and GA areas. The MEFOS fields cover the regions of highest galaxy density, with galaxy densities typically in excess of 25 galaxies per square degree (see lower panel of Fig. 1).

\subsection{Data reduction}

All the MEFOS spectra have been reduced with the standard IRAF $^{1}$ software package. Various command language (cl) programmes were written by V. Cayatte which greatly streamlined the reduction process. This process involved the extraction of the individual spectra, the wavelength calibration using helium and neon lamp arc exposures, a correction for the fibre transmission, the sky subtraction, the removal of cosmic rays and the determination of the redshift.

Thirteen helium and neon emission lines were used for the wavelength calibration. The wavelength solution to the fit of the arc lines was accurate to better than $\sim 0.3 \AA$. A number of fibres showed a significant offset in the position of the [OI] skyline with respect to its nominal value of $5577.35 \AA$, in some cases as much as $1.8 \pm 0.1 \AA$ (Felenbok et al. 1997, but see also Batuski et al. 1999). These offsets are systematic and are caused by a misalignment of some of the fibres with respect to the slit. For those fibres where this shift is larger than $0.5 \AA$, an inverse wavelength shift of the average offset (averaged over the entire observing run) to the fibre in question was applied.

The relative fibre transmission coefficient for each fibre is determined from the [O I] $5577.35 \AA$ A skyline flux. For each MEFOS field the measured flux is normalised using the fibre with the highest throughput. The transmission might vary from night to night due to sky variations or changes in the fibre efficiency (Felenbok et al. 1997). The first effect is rather weak below $6000 \AA$ (Cuby \& Magnoli 1994) and as we find no evidence for variations in the overall transmission coefficient (the rootmean-square error is $1-3 \%$ ) the latter effect is not significant.

However, a clear drop in the fibre transmission coefficients between the 1994 and 1995 data was seen in 6 of the 30 fibres. In a time span of more than one year some fibres had lost as much as $30 \%$ of their efficiency. The likely cause for this drop in efficiency is physical damage to the fibres in question, as they are not expected to have any natural decay.

After the fibre transmission corrections were made, the sky spectra within each MEFOS field were examined by eye. The sky spectra were averaged to reduce the noise in the skysubtracted spectrum.

\subsection{Redshift determination}

The IRAF package RVSAO was used to determine the redshifts. Four template stars have been observed; they form a template for the cross-correlation of galaxy spectra. The actual cross-correlation of the spectra is done with XCSAO within

\footnotetext{
${ }^{1}$ IRAF (Image Reduction and Analysis Facility) is distributed by the National Optical Astronomy Observatories, which are operated by the Association of Universities for Research in Astronomy, Inc., under cooperative agreement with the National Science Foundation.
} 


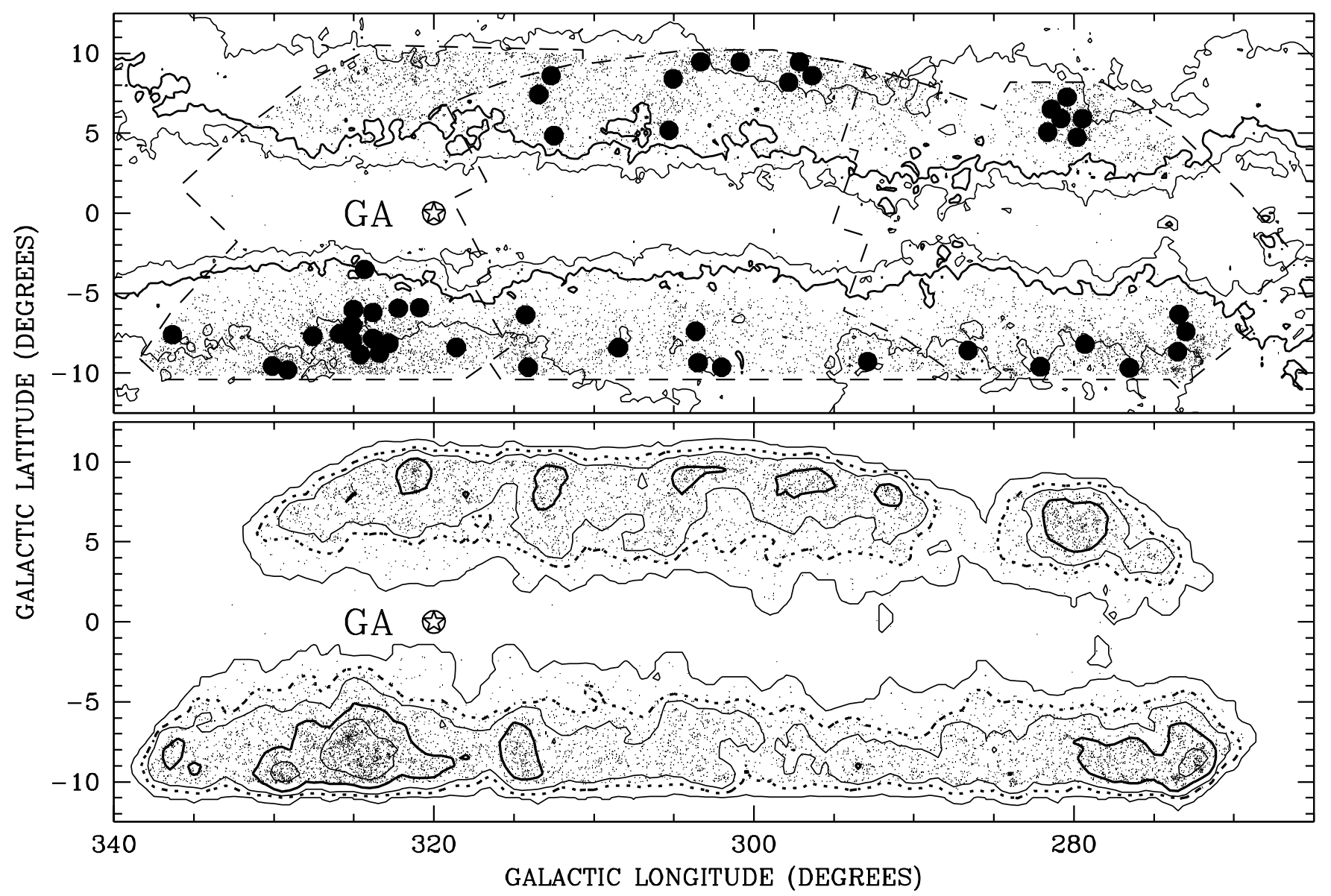

Fig. 1. The distribution of galaxies in the Hydra/Antlia, Crux and GA region (upper panel, from right to left). The dashed line marks these three different survey regions. The contours are lines of equal Galactic foreground extinction, taken from the Galactic reddening maps of Schlegel et al. (1998). The contours correspond to $A_{\mathrm{B}}=1^{\mathrm{m}}, 3^{\mathrm{m}}$ (thick line) and $5^{\mathrm{m}}$. In the upper panel, the big dots show the location of the 48 observed MEFOS fields. The lower panel shows the galaxy density contours in the Hydra/Antlia, Crux and GA region. The contours mark $0.5,5$ (dotted line), 10, 25 (thick line) and 50 galaxies per square degree, respectively. The position of the peak of the reconstructed mass density field associated with the GA (Kolatt et al. 1995) is clearly marked.

the RVSAO package. After a first cross-correlation (with no a priori imposed velocity limits), cross-correlation peaks were checked by eye for each galaxy. Galaxies with an erroneous cross-correlation peak of low contrast were either rejected, or, when a clear (unambiguous) peak was seen elsewhere with matching $\mathrm{H}$ and $\mathrm{K}$ calcium lines, remained in the final list of galaxies.

The galaxies in this list were then cross-correlated one more time, but now with a strict velocity range imposed. For galaxies with redshifts lower than $5000 \mathrm{~km} \mathrm{~s}^{-1}$ this range is $\pm 1000 \mathrm{~km} \mathrm{~s}^{-1}$, for more distant galaxies the velocity window ranges from $0.85 \times v_{\text {obs }}$ to $1.15 \times v_{\text {obs }}$.

Emission line galaxies were treated separately. Their redshifts were determined via the EMSAO task within the RVSAO package. The errors in the quoted MEFOS emission line redshifts are conforming to the errors in the SAAO data (Papers I-III), i.e., $100 \mathrm{~km} \mathrm{~s}^{-1}$ in case of a single emission line and $100 / \sqrt{N}$ in case of $N$ emission lines.

The errors in the quoted MEFOS redshifts from the absorption lines are not true "external" errors. They are based on an internal comparison, rather than a comparison with literature data. One field has been observed twice (field F18) resulting in 16 galaxies having two independently measured MEFOS redshifts. A further 4 galaxies were found on overlapping fields. For these 20 galaxies, the difference between the two redshift measurements is plotted (as crosses) in Fig. 2 against the Tonry $\&$ Davies (1979) $R$-parameter.

The $\sigma_{Q}$ method (Hill \& Oegerle 1993; Pinkney et al. 1993) is often used to infer an error $\left(\sigma_{Q}=Q /(R+1)\right)$ on the basis of the observed $R$-parameter (contrast factor). Based on the 20 overlapping galaxies displayed in Fig. 2, $Q_{\text {MEFOS }}=$ $315 \mathrm{~km} \mathrm{~s}^{-1}$ corresponds best to the uncertainties in our MEFOS data, i.e., the solid line drawn in Fig. 2. This corresponds reasonably well with the $Q$-value obtained by Batuski et al. (1999) from their MEFOS observations.

The solid dots in Fig. 2 are the errors as quoted in our redshift catalogue. These errors correspond to twice the standard deviation of the $R$-weighted average velocity. These errors, rather than $\sigma_{Q}$, were used in our redshift catalogue. They are, as can be seen in Fig. 2, entirely consistent with $Q=315 \mathrm{~km} \mathrm{~s}^{-1}$. 


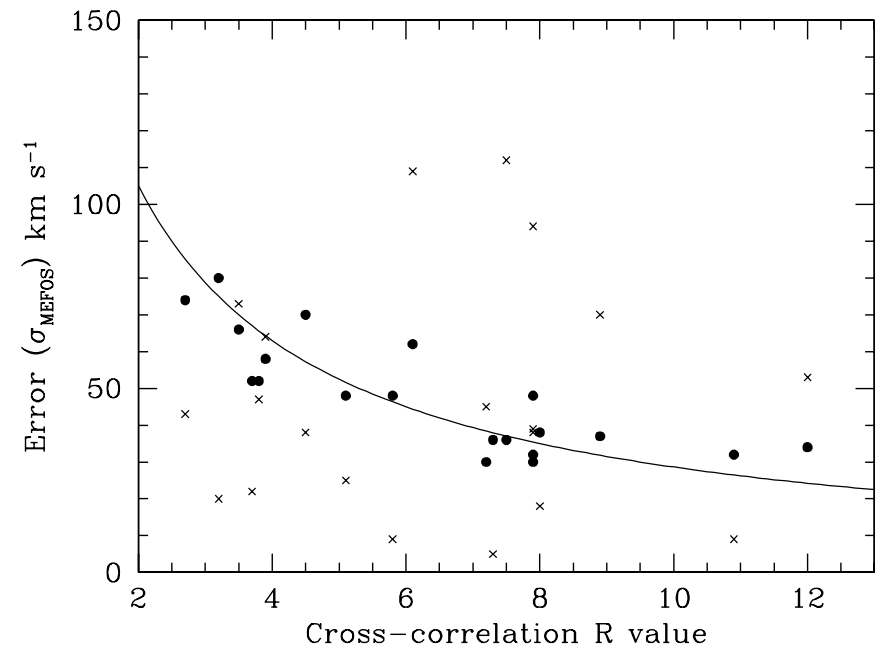

Fig. 2. The external error in the MEFOS redshifts, as a function of the $R$-parameter. The crosses represent the difference in velocity between two independent measurements, the filled circles correspond to the errors used by us. The curve shows global uncertainties according to the $\sigma_{Q}$ method, with $Q=315 \mathrm{~km} \mathrm{~s}^{-1}$.

\subsection{The redshift catalogue}

The 764 redshifts obtained with MEFOS are presented in Table $1^{2}$. The entries in Table 1 are as follows:

Columns 1 and 2: identification of the galaxy as given in Kraan-Korteweg (2000) - prefix "RKK" - and Woudt \& Kraan-Korteweg (2001) - prefix "WKK" -, and Lauberts identification (Lauberts 1982).

Columns 3 and 4: Right Ascension and Declination (2000.0). The positions were measured with the Optronics machine at ESO in Garching and have an accuracy of about 1 arcsec.

Columns 5 and 6: galactic longitude $\ell$ and latitude $b$.

Column 7: major and minor axes (in arcsec). These diameters are measured approximately to the isophote of $24.5 \mathrm{mag} \operatorname{arcsec}^{-2}$ and have a scatter of $\sigma \approx 4^{\prime \prime}$.

Column 8: apparent magnitude $B_{25}$. The magnitudes are estimates from the film copies of the SRC IIIaJ Survey based on the above given diameters and an estimate of the average surface brightness of the galaxy.

Column 9: morphological type. The morphological types are coded similarly to the precepts of the Second Reference Catalogue (de Vaucouleurs et al. 1976). Due to the varying foreground extinction a homogeneous and detailed type classification could not always be accomplished and some codes were added: in the first column $\mathrm{F}$ for $\mathrm{E} / \mathrm{S} 0$ was added to the normal designations of E, L, S and I. In the fourth column the subtypes $\mathrm{E}, \mathrm{M}$ and $\mathrm{L}$ are introduced next to the general subtypes 0 to 9 . They stand for early spiral (S0/a$\mathrm{Sab})$, middle spiral ( $\mathrm{Sb}-\mathrm{Sd})$ and late spiral (Sdm-Im). The cruder subtypes are a direct indication of the fewer details visible in the obscured galaxy image. The question mark at

\footnotetext{
2 Table 1 is only available in electronic form at the CDS via anonymous ftp to cdsarc.u-strasbg.fr (130.79.128.5) or via http: //cdsweb.u-strasbg.fr/cgi-bin/qcat? J/A+A/415/9
}

the end marks uncertainty of the main type, the colon marks uncertainty in the subtype.

Column 10: heliocentric velocity $(c z)$ and error as derived from the absorption features. The errors may appear large as they are estimated external errors, and not internal errors (see Sect. 2.2). The square brackets indicate a tentative redshift.

Column 11: heliocentric velocity and error measured from the emission lines (identified in Col. 12) when present. The square brackets indicate a tentative redshift.

Column 12: code for the emission lines identified in the respective spectra:

$\begin{array}{ccccc}1 & 2 & 3 & 4 & 5 \\ {[\mathrm{OII}]} & \mathrm{H} \gamma & \mathrm{H} \beta & {[\mathrm{OIII}]} & {[\mathrm{OIII}]} \\ 3727 & 4340 & 4861 & 4959 & 5007\end{array}$

Column 13: code for additional remarks:

* - Redshift is also available in the literature (NASA Extragalactic Database (NED), May 2003).

1 - RKK 1808: the redshift measured with MEFOS is in slight disagreement with the value quoted in the literature $\left(v=5571 \pm 150 \mathrm{~km} \mathrm{~s}^{-1}\right.$, Paper I). Preference is given to the value quoted here.

2 - RKK 2313: the redshift measured with MEFOS is in slight disagreement with the (tentative) value quoted in the literature $\left(v=5741 \pm 300 \mathrm{~km} \mathrm{~s}^{-1}\right.$, Paper I). Preference is given to the value quoted here.

3 - WKK 662: the redshift measured with MEFOS is in slight disagreement with the (tentative) value quoted in the literature $\left(v=6526 \pm 231 \mathrm{~km} \mathrm{~s}^{-1}\right.$, Paper II). Preference is given to the value quoted here.

4-WKK 708: the redshift measured with MEFOS is in disagreement with the (tentative) value quoted in the literature $\left(v=1326 \pm 243 \mathrm{~km} \mathrm{~s}^{-1}\right.$, Paper II). The clear emission lines in the MEFOS spectra support the rejection of the literature value.

5 - WKK 1883: the redshift quoted in the literature $(v=$ $6801 \pm 70 \mathrm{~km} \mathrm{~s}^{-1}$, Visvanathan \& Yamada 1996) is in disagreement with the redshift determined from the MEFOS spectra. The latter shows a clear range of absorption lines and its redshift determination is unambiguous. Preference is given to the MEFOS value.

6 - WKK 5094: the redshift measured with MEFOS is in disagreement with the (tentative) value quoted in the literature $\left(v=17956 \pm 250 \mathrm{~km} \mathrm{~s}^{-1}\right.$, Paper III). Preference is given to the value quoted here.

7 - WKK 5366: the redshift quoted in the literature $(v=$ $2059 \pm 10 \mathrm{~km} \mathrm{~s}^{-1}$, Juraszek et al. 2000) is incompatible with the MEFOS redshift. There is a galaxy (WKK 5365) $\sim 3.5^{\prime}$ from WKK 5366, and the literature value (H I line spectroscopy) could have suffered from source confusion.

8 - WKK 5416: the redshift quoted in the literature $(v=$ $12403 \pm 70 \mathrm{~km} \mathrm{~s}^{-1}$, Visvanathan \& Yamada 1996) is in disagreement with the redshift determined from the MEFOS spectra. The latter shows a clear range of absorption lines 


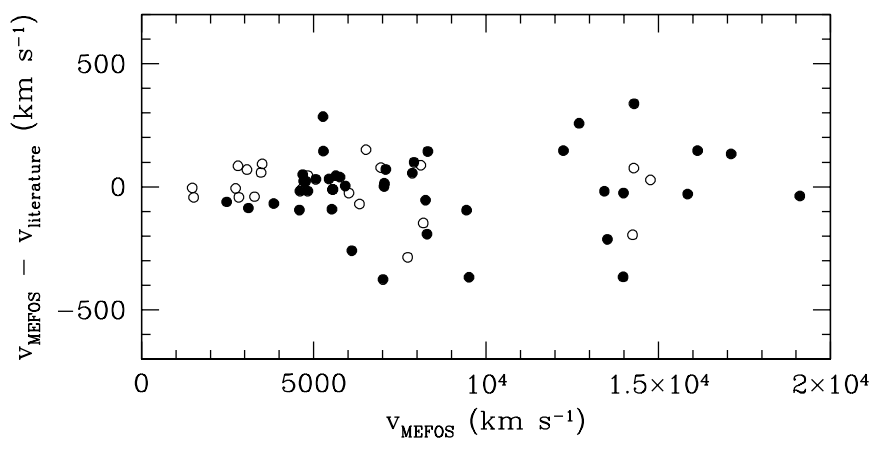

Fig. 3. The velocity difference $\left(v_{\mathrm{MEFOS}}-v_{\text {literature }}\right)$ as a function

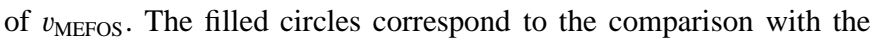
SAAO data (Papers I-III), the open circles correspond to the remainder of the literature sample.

and its redshift determination is unambiguous. Preference is given to the value quoted here.

In Table $2^{3}$, we present the 183 galaxies observed with MEFOS, but for which no redshift could be obtained due to the presence of superimposed foreground stars on the galaxy, or due to the low signal-to-noise of the resulting spectra. The entries in Table 2 are as follows:

Columns 1-8: as in Table 1.

Column 9: as in Table 1. In addition, a question mark prior to the galaxy classification indicates uncertainty about the galaxian nature of the candidate.

Column 10: remarks about the quality of the spectrum.

\subsection{Comparison to other measurements}

There are 71 galaxies in Table 1 for which a redshift has been obtained previously (NED, May 2003). For 8 of them, the value quoted here is in disagreement with the literature data (see Col. 13 of Table 1), but for the remaining 63 galaxies there is good agreement. We find that

$<v_{\text {MEFOS }}-v_{\text {literature }}>=-8 \pm 18 \mathrm{~km} \mathrm{~s}^{-1}$.

If the overlapping sample is restricted to measurements made by us at the SAAO (Papers I-III), the agreement again is good, although the standard deviation is somewhat larger

$<v_{\text {MEFOS }}-v_{\text {SAAO }}>=-9 \pm 24 \mathrm{~km} \mathrm{~s}^{-1}$,

based on 42 galaxies in common. The larger standard deviation is due to the increasing uncertainty in the SAAO spectra of the more distant galaxies. Figure 3 shows the general good agreement between the MEFOS redshifts and the literature values.

\section{Coverage and completeness}

In the following two sections, only the galaxies with a reliable redshift $(N=754)$ will be used in the discussion and plots; the galaxies for which we obtained a tentative redshift $(N=10)$ will not be included.

${ }_{3}$ Table 2 is only available in electronic format at the CDS via anonymous ftp to cdsarc.u-strasbg.fr $(130.79 .128 .5)$ or via http://cdsweb.u-strasbg.fr/cgi-bin/qcat?J/A+A/415/9

\subsection{The Hydra/Antlia region}

The Hydra/Antlia region contains 3279 galaxy candidates (Kraan-Korteweg 2000). 183 Galaxies in this region have a previously determined redshift (NED, and references therein). Most of these redshifts were determined in the course of our redshift survey (Kraan-Korteweg et al. 1995; Kraan-Korteweg et al. 2002).

The MEFOS observations have resulted in 185 redshifts in the Hydra/Antlia region (see Table 1), of which 7 are tentative measurements. For eighteen galaxies, a redshift had been published previously, so 160 new redshifts in the Hydra/Antlia region are presented here. A total of 343 galaxies (literature + MEFOS) in the Hydra/Antlia region $(=10.5 \%)$ now have a reliable redshift. This percentage is somewhat lower than that in the Crux and GA regions (see Sects. 3.2 and 3.3), but additional spectra have been obtained for 500 galaxies with Optopus (Kraan-Korteweg et al. 1994), and will be presented elsewhere.

The upper-left panel of Fig. 4 shows the magnitude distribution $\left(B_{25}\right)$ of all the 343 galaxies with a reliable redshift (cross-hatched histogram). The filled histogram shows the magnitude distribution of the 178 galaxies with a reliable MEFOS redshift. It is clear that the MEFOS observations trace the fainter end of the magnitude distribution of the galaxies in the Hydra/Antlia region. The average $B_{25}$ of the MEFOS sample is 17.9 , only slightly brighter than the overall mean magnitude of the galaxies in the Hydra/Antlia catalogue, 18.2 (Kraan-Korteweg 2000).

The mean extinction-corrected magnitude of the MEFOS sample is $\left\langle B_{25}^{0}>=16^{\mathrm{m}} \cdot 6\right.$, compared to $<B_{25}^{0}>=15$. 6 for the entire redshift sample (literature and MEFOS). We have used the Galactic reddening maps of Schlegel et al. (1998) for the extinction correction; see Woudt \& Kraan-Korteweg (2001) for full details of the extinction correction procedure.

\subsection{The Crux region}

In the Crux region, 3759 galaxy candidates have been identified (Woudt \& Kraan-Korteweg 2001), and 270 of them have a reliable redshift reported in the literature. The MEFOS observations have resulted in 251 galaxy redshifts in the Crux region (see Table 1), of which 2 were tentative measurements. Fifteen galaxies of the MEFOS sample had a previous redshift measurement, so 234 new reliable redshifts have been obtained. This increases the total number of galaxies in the Crux region with a redshift to 504 (13.4\%).

As before in the Hydra/Antlia region, the MEFOS sample contributes predominantly to the faint end of the galaxy distribution (upper-middle panel of Fig. 4). The mean magnitude of the 249 galaxies MEFOS sample is $\left\langle B_{25}\right\rangle=18$ m 0 , again only slightly brighter than the overall mean magnitude of all the galaxies in the Crux region $\left(\left\langle B_{25}\right\rangle=18\right.$. 2 , Woudt \& Kraan-Korteweg 2001).

\subsection{The Great Attractor region}

4423 Galaxy candidates have been identified in the Great Attractor region (Woudt \& Kraan-Korteweg 2001), 413 of them 


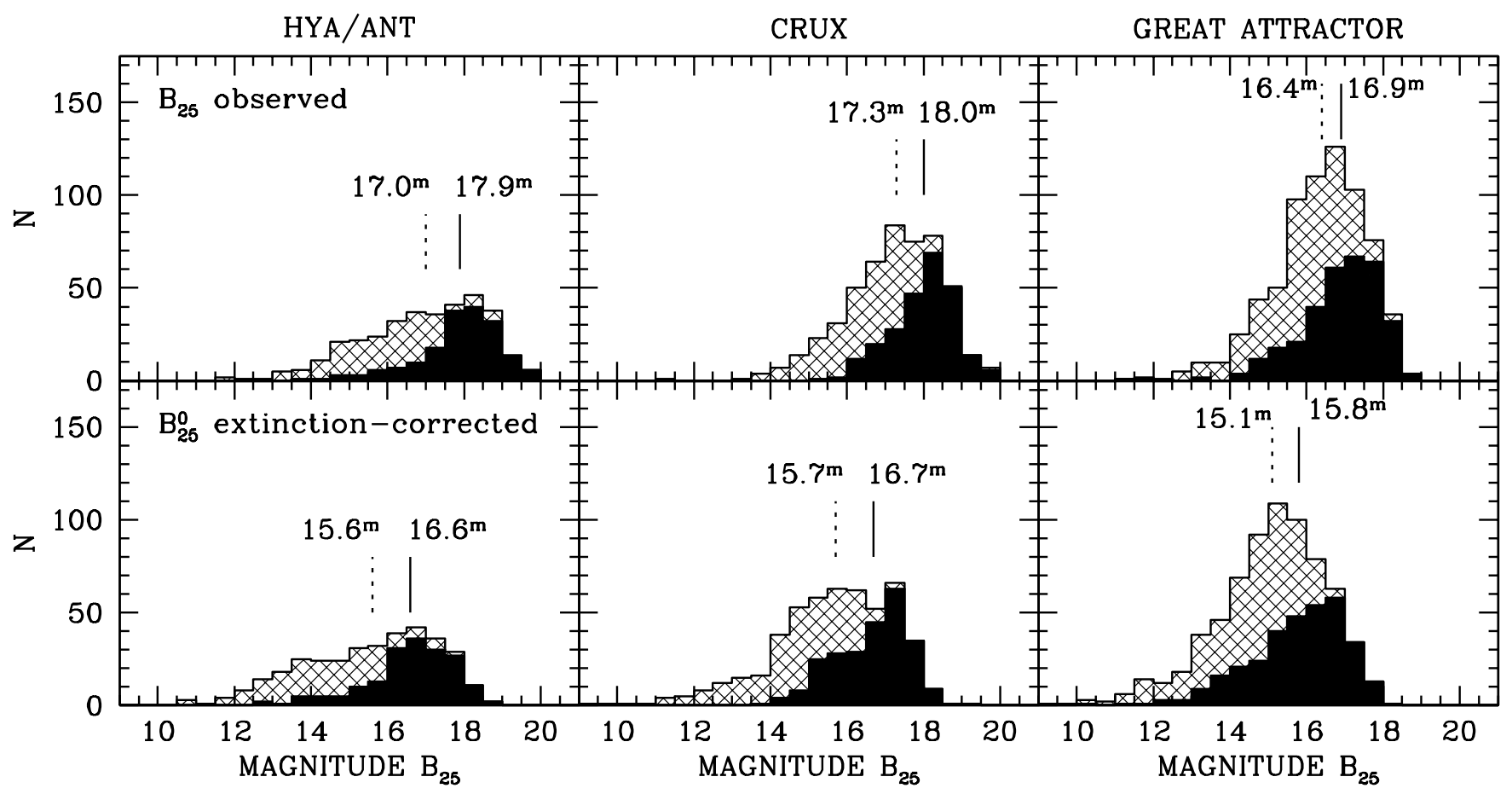

Fig. 4. The upper row of panels show the magnitude $\left(B_{25}\right)$ distribution of galaxies in the combined (literature + MEFOS) sample (cross-hatched histogram) and MEFOS sample alone (black histogram), for the Hydra/Antlia (left), Crux (middle) and GA region (right), respectively. The lower row of panels show the extinction-corrected magnitude $\left(B_{25}^{0}\right)$ distribution. The symbols are the same as the upper row of panels. The mean values for the combined, and MEFOS sample are indicated by the dashed, and solid vertical line for the combined (literature + MEFOS), and MEFOS sample, respectively.

have a reliable redshift (NED, May 2003). The Norma cluster (ACO 3627, Abell et al. 1989) is the region of highest galaxy density in the GA region, and as a result a large number of the MEFOS fields were centred on the Norma cluster (see also the lower panel of Fig. 1). We have obtained 328 redshifts in the GA region, one of which is a tentative measurement. Of the remaining 327 galaxies, 38 had a previously published redshift, therefore the MEFOS spectroscopy has resulted in 289 new galaxy redshifts in the GA region. A total of 702 galaxies $(15.9 \%)$ of all the galaxies in the GA region now have a redshift (literature + MEFOS).

The mean brightness of the 327 galaxies in the MEFOS sample is $\left\langle B_{25}\right\rangle=16$. 9 , about 1 magnitude brighter than the MEFOS sample in the Hydra/Antlia and Crux regions. This is due to the presence of a nearby overdensity - the rich Norma cluster (Kraan-Korteweg et al. 1996) - in the GA region, whereas most of the regions of high galaxy density in the Hydra/Antlia and Crux region correspond to more distant clusters (see Sect. 4).

\subsubsection{The Norma cluster}

Within the Abell radius of the Norma cluster (defined as $3 h_{50}^{-1} \mathrm{Mpc}$ ), 603 galaxies are present in our galaxy catalogue (Woudt \& Kraan-Korteweg 2001). We have now obtained redshifts for 265 of them $(=44 \%)-130$ of these were obtained with MEFOS. Of the 265 galaxies with a reliable redshift, 214 are comfirmed cluster members. The others are clear foreground/background galaxies. Prior to our redshift survey, only 17 redshifts were known within the Abell radius of the Norma cluster.

For galaxies with $B_{25}^{0} \leq 15.5,80 \%$ now have a reliable redshift. Most of the missing bright galaxies are low surface brightness spirals, which have been observed with the Parkes 64-m radio telescope and will be presented elsewhere.

A detailed dynamical analysis of the Norma cluster will be presented elsewhere (including recently obtained $2 \mathrm{dF}$ spectroscopy of the Norma cluster). It is clear, however, that the MEFOS observations form an essential basis for this analysis.

\section{Identification of large-scale structures}

\subsection{Velocity distribution}

The velocity histograms in Fig. 5 show that distinctly different large-scale structures are present in the Hydra/Antlia, Crux and $\mathrm{GA}$ region, respectively.

The Hydra/Antlia region shows a strong peak at $v \sim$ $2750 \mathrm{~km} \mathrm{~s}^{-1}$, which corresponds to the extension of the Hydra/Antlia supercluster in to the Zone of Avoidance (Paper I; Kraan-Korteweg 2000). In addition, an overdensity at $\sim 6000 \mathrm{~km} \mathrm{~s}^{-1}$ is present, associated with the Vela overdensity (Paper I). The MEFOS spectra have not added much information to the nearby structures $\left(<10000 \mathrm{~km} \mathrm{~s}^{-1}\right)$, but instead have unveiled more distant galaxies. Most of the redshifts of galaxies beyond $10000 \mathrm{~km} \mathrm{~s}^{-1}$ have come from the MEFOS spectroscopy. In the Hydra/Antlia region, there are 16 galaxies with 


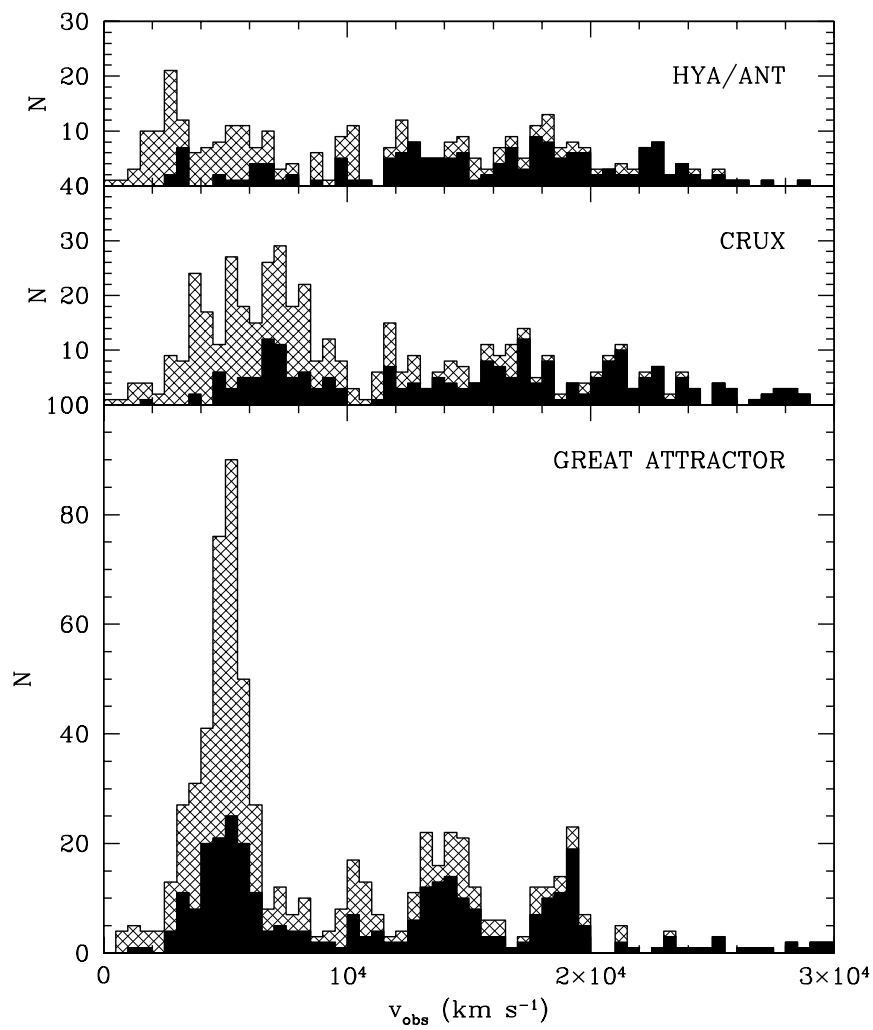

Fig. 5. Velocity histogram of the galaxies in the Hydra/Antlia region (upper panel), the Crux region (middle panel) and the GA region (lower panel). The dark shaded area correspond to the heliocentric velocities obtained with MEFOS, the cross-hatched region shows all the heliocentric velocities available to date.

heliocentric velocities in excess of $30000 \mathrm{~km} \mathrm{~s}^{-1}$ (not shown in Fig. 5). These are all obtained with MEFOS.

In the Crux region, a broad concentration of galaxies around $3500<v<8500 \mathrm{~km} \mathrm{~s}^{-1}$ is present (middle panel of Fig. 5). This broad concentration - a combination of the Centaurus-Crux cluster (Woudt 1998, also known as CIZA J1324.7-5736, Ebeling et al. 2002) and the large-scale overdensity between the Norma cluster and the Vela cluster (see also panel C of Fig. 6) - is very different from the distinct peaks at $2750 \mathrm{~km} \mathrm{~s}^{-1}$ and $5000 \mathrm{~km} \mathrm{~s}^{-1}$, in the Hydra/Antlia and Great Attractor region, respectively. Again, the MEFOS observations trace the more distant galaxies more efficiently compared to the earlier SAAO data (Papers I-III). In the Crux region, 32 galaxies are located beyond $30000 \mathrm{~km} \mathrm{~s}^{-1}$. Again, all these redshifts were obtained with MEFOS.

The GA region is dominated by four distinct peaks, the most prominent of which is associated with the Norma cluster and its surrounding great wall-like structure at $4848 \pm$ $61 \mathrm{~km} \mathrm{~s}^{-1}$ (Kraan-Korteweg et al. 1996; Woudt 1998). The other peaks are at $10406 \pm 82 \mathrm{~km} \mathrm{~s}^{-1}, 14050 \pm 84 \mathrm{~km} \mathrm{~s}^{-1}$ and $18689 \pm 81 \mathrm{~km} \mathrm{~s}^{-1}$. The peak at $14050 \mathrm{~km} \mathrm{~s}^{-1}$ belongs to the Ara cluster (Woudt 1998; Paper III; Ebeling et al. 2002). Together with the adjacent Triangulum-Australis cluster (McHardy et al. 1981), the Ara cluster is part of a larger overdensity referred to as a "Greater Attractor behind the Great Attractor" by Saunders et al. (2000). Saunders et al. find evidence for such an overdensity at $\sim 12500 \mathrm{~km} \mathrm{~s}^{-1}$ and $(\ell, b) \approx\left(326^{\circ},-3^{\circ}\right)$ from the reconstructed IRAS galaxy density field. In the GA region there are 18 galaxies more distant than $30000 \mathrm{~km} \mathrm{~s}^{-1}$, all derived from MEFOS observations.

\subsection{Sky projection; local cosmography}

In Fig. 6, all the galaxies out to $16000 \mathrm{~km} \mathrm{~s}^{-1}$ are shown in an Aitoff projection of a limited area of the sky $\left(240^{\circ} \leq \ell<\right.$ $360^{\circ},-30^{\circ}<b<+30^{\circ}$ ). They are separated in eight individual slices, each $2000 \mathrm{~km} \mathrm{~s}^{-1}$ thick, but within each panel the different symbols mark a subdivision: the filled symbols show the "nearer" $1000 \mathrm{~km} \mathrm{~s}^{-1}$ (e.g. 0-1000 $\mathrm{km} \mathrm{s}^{-1}$ in the topleft panel), the open symbols the "further" $1000 \mathrm{~km} \mathrm{~s}^{-1}$ (e.g. $1000-2000 \mathrm{~km} \mathrm{~s}^{-1}$ in the top-left panel). The data shown are literature values (small squares: NED, May 2003) combined with the MEFOS heliocentric velocities (big circles). The Aitoff projections are centred on $\ell=300^{\circ}$ and $b=0^{\circ}$.

Here, we will discuss the unveiled large-scale structures behind the southern Milky Way out to $16000 \mathrm{~km} \mathrm{~s}^{-1}$, in relation to the structures visible away from the Galactic Plane (GP).

$v \leq 2000 \mathrm{~km} \mathrm{~s}^{-1}$ : the Supergalactic Plane (SGP) is visible as a concentration of galaxies starting from the Virgo cluster (located outside this panel at $\left.(\ell, b)=\left(280^{\circ},+74^{\circ}\right)\right)$, crossing the GP at $\ell=325^{\circ}$, and continuing down towards the south Galactic pole. Also, the Fornax wall is discernable in the 1000$2000 \mathrm{~km} \mathrm{~s}^{-1}$ range as an overdensity of galaxies starting from the Fornax cluster at $(\ell, b)=\left(237^{\circ},-54^{\circ}\right)$ towards (and crossing) the GP at $\ell=290^{\circ}$. The GA overdensity is dominant in the next two slices.

$2000<v \leq 4000 \mathrm{~km} \mathrm{~s}^{-1}$ : the vertical band of galaxies associated with the SGP in the previous slice is very pronounced in this redshift slice (and the next one); this is the Centaurus wall, a very extended structure (both on the sky, as in depth), incorporation both the Centaurus cluster $(\ell, b)=\left(302^{\circ},+22^{\circ}\right)$ and the Norma cluster $(\ell, b)=\left(325^{\circ},-7^{\circ}\right)$. The Norma cluster is visible in this panel, but is most pronounced in the range $4000-6000 \mathrm{~km} \mathrm{~s}^{-1}$. A wall-like structure is also visible crossing the GP at $\ell=240^{\circ}$. This is the Hydra wall, encompassing the Hydra cluster $(\ell, b)=\left(270^{\circ},+27^{\circ}\right)$ and the Puppis cluster $(\ell, b)=\left(240^{\circ}, 0^{\circ}\right)$, and continuing towards $(\ell, b)=\left(210^{\circ},-30^{\circ}\right)$.

$4000<v \leq 6000 \mathrm{~km} \mathrm{~s}^{-1}$ : a large structure of galaxies is seen below the GP extending from $(\ell, b) \approx\left(30^{\circ},-60^{\circ}\right)-10-$ cated outside the boundary of this plot - to the Norma cluster. This structure, which is part of the Norma supercluster, is under a slight angle with respect to the Centaurus wall in the previous two slices. Here, the Norma supercluster and the Centaurus wall possibly intersect. Also, parts of the Norma supercluster can be seen (in the more distant 5000-6000 $\mathrm{km} \mathrm{s}^{-1}$ range - north of the GP around $\ell=280^{\circ}-315^{\circ}$, incorporating the Vela cluster (Kraan-Korteweg \& Woudt 1994) and the Centaurus-Crux cluster (Woudt 1998). From X-ray observations, the latter (CIZA J1324.7-5736) appears possibly as massive as the Norma cluster (Ebeling et al. 2002).

$6000<v \leq 8000 \mathrm{~km} \mathrm{~s}^{-1}$ : the finger-of-god effect for both the Norma cluster and CIZA J1324.7-5736 cluster appear in 

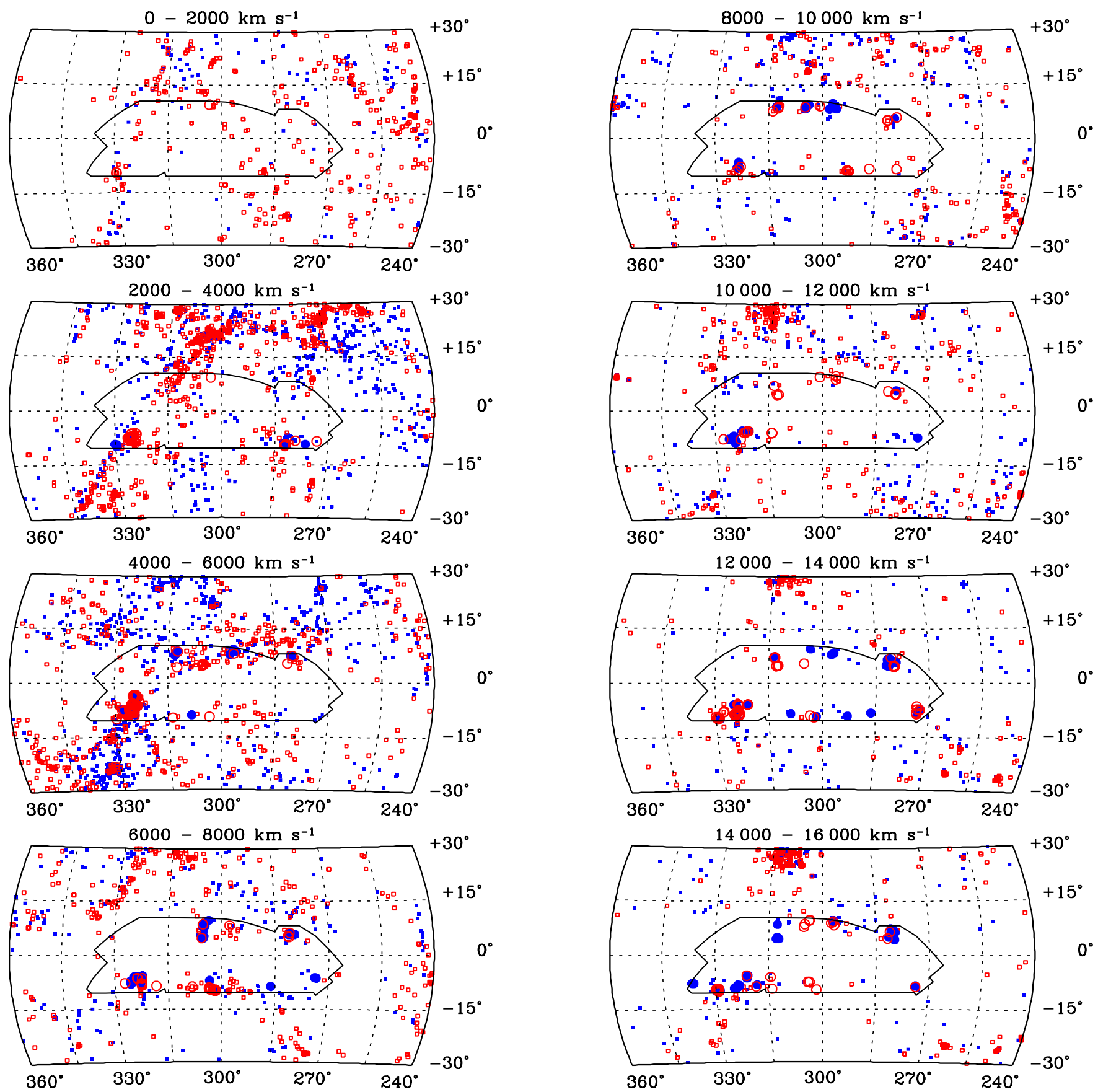

Fig. 6. An Aitoff projection of galaxies in the range of $240^{\circ} \leq \ell<360^{\circ}$ and $-30^{\circ}<b<+30^{\circ}$, in heliocentric velocity intervals of $\Delta v=$ $2000 \mathrm{~km} \mathrm{~s}^{-1}$. These graphs are centred on $\ell=300^{\circ}$ and $b=0^{\circ}$ with decreasing longitudes towards the right. Our search area is marked and the velocity range is indicated on the top of each panel. Within each panel, the filled symbols (small squares: NED, big circles: our MEFOS data) show the nearer $1000 \mathrm{~km} \mathrm{~s}^{-1}$ slice, the open symbols (again, small squares: NED, big circles: our MEFOS data) show the more distant $1000 \mathrm{~km} \mathrm{~s}^{-1}$ slice.

this panel. In this velocity range, few, or none, extented structures are seen in the southern sky.

$8000<v \leq 10000 \mathrm{~km} \mathrm{~s}^{-1}$ : there is a slight overdensity north of the Galactic plane at $\ell \approx 295^{\circ}$ in the nearer range (filled circles), but no distinct clusters are visible in our surveyed region. Note, however, the Ophiuchus cluster (Hasegawa et al. 2000) at the edge of the plot $(\ell, b, v) \approx\left(360^{\circ},+9^{\circ}\right.$, $8500 \mathrm{~km} \mathrm{~s}^{-1}$ ). $10000<v \leq 12000 \mathrm{~km} \mathrm{~s}^{-1}$ : the Shapley region comes into view at $(\ell, b) \approx\left(315^{\circ},+25^{\circ}\right)$. Behind the Norma cluster $\left(\ell \approx 325^{\circ}\right)$ an overdensity is visible (continuing into the next panel), corresponding to the peak in Fig. 5 at $10500 \mathrm{~km} \mathrm{~s}^{-1}$.

$12000<v \leq 16000 \mathrm{~km} \mathrm{~s}^{-1}:$ in these more distant slices, the Shapley region dominates the galaxy distribution above the $\mathrm{GP}$, and within our surveyed region various clusters are visible (see also the pie diagrams shown in Fig. 7). The most massive 


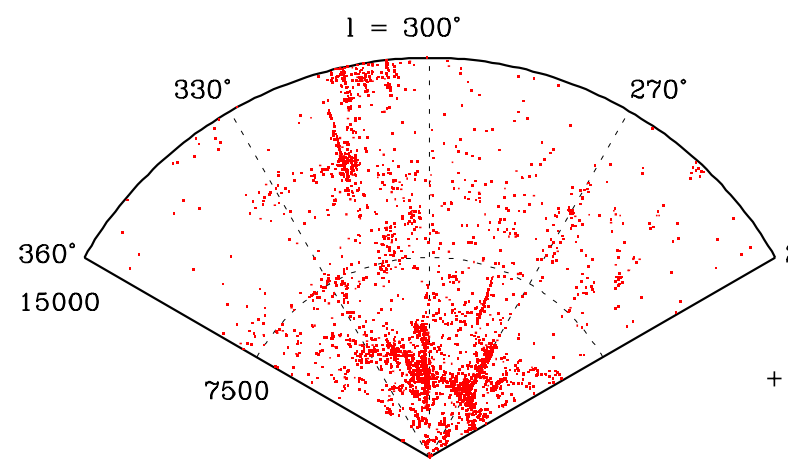

$240^{\circ}$

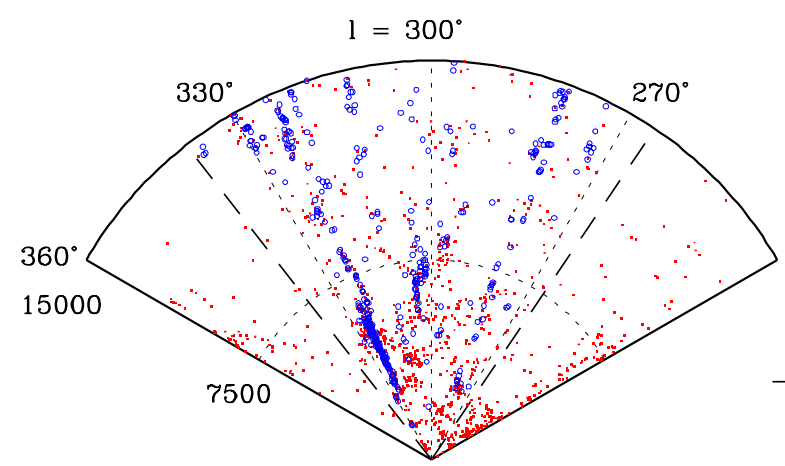

$+10^{\circ} \leqq \mathrm{b}<+30^{\circ}$

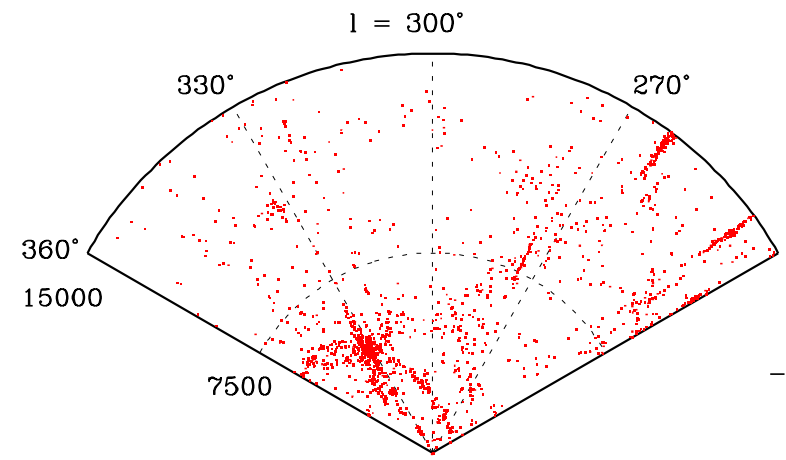

$240^{\circ}$

$-30^{\circ}<\mathrm{b} \leqq-10^{\circ}$

$240^{\circ}$

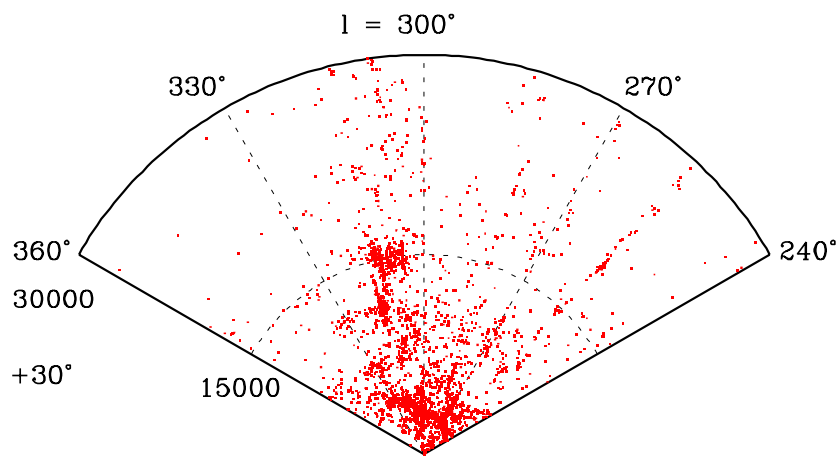

$1=300^{\circ}$

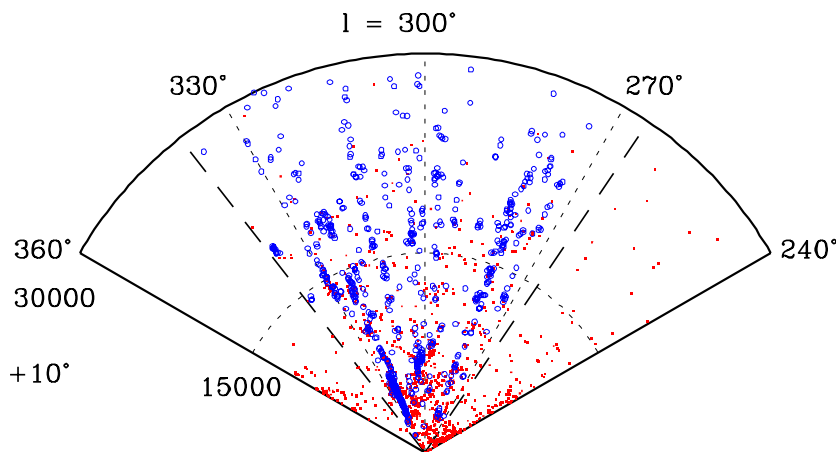

Fig. 7. Redshift slices out to $v<15000 \mathrm{~km} \mathrm{~s}^{-1}$ (left column) and $v<30000 \mathrm{~km} \mathrm{~s}^{-1}$ (right column) for the longitude range $240^{\circ} \leq \ell<360^{\circ}$. The top panels display the structures above the Galactic Plane $\left(+10^{\circ} \leq b<+30^{\circ}\right)$, the middle panels show the structures behind the Galactic Plane $\left(-10^{\circ}<b<+10^{\circ}\right)$, and the lower panels show the large-scale structures at $-30^{\circ}<b \leq-10^{\circ}$. The dashed lines in the middle panels show the demarcation of our survey area. The data shown are literature values (small dots: NED, May 2003) combined with the MEFOS heliocentric velocities (open circles).

cluster is the Ara cluster (see also Sect. 4.1.) at $(\ell, b, v, \sigma) \approx$ $\left(329.4^{\circ},-9.3^{\circ}, 14722 \mathrm{~km} \mathrm{~s}^{-1}, 1182 \mathrm{~km} \mathrm{~s}^{-1}\right)$.

\subsection{Pie diagrams}

An alternative view of the large-scale structures in this part of the sky $\left(240^{\circ} \leq \ell<360^{\circ},|b|<30^{\circ}\right)$ is given by the pie diagrams in Fig. 7. They show the galaxy distribution out to $15000 \mathrm{~km} \mathrm{~s}^{-1}$ (left panel), and $30000 \mathrm{~km} \mathrm{~s}^{-1}$ (right panel).

In this representation, the Zone of Avoidance is almost indistinguishable from its neighbouring (unobscured) region above and below the GP. In the Zone of Avoidance out to $30000 \mathrm{~km} \mathrm{~s}^{-1}$ (middle right panel of Fig. 7), there are 1857 galaxies. In the adjacent region below the Galactic Plane (lower right panel), 1981 galaxies are present; an almost identical number of galaxies. Above the GP more galaxies are present $(N=3497)$. Note, however, that most of the redshifts in the Zone of Avoidance are for galaxies in the Galactic latitude range of $|b| \sim 5^{\circ}-10^{\circ}$. It is remarkable, nonetheless, that this region of the sky that was previously devoid of information, now shows clear clusters, superclusters and voids.

In the galaxy distribution out to $15000 \mathrm{~km} \mathrm{~s}^{-1}$ (middle left panel $)$, the Norma cluster at $(\ell, v)=\left(325.3^{\circ}, 4844 \mathrm{~km} \mathrm{~s}^{-1}\right)$ dominates the pie diagram. It is located centrally in an overdensity that connects the Centaurus cluster $(\ell, v) \approx\left(302^{\circ}, 3500 \mathrm{~km} \mathrm{~s}^{-1}\right)$ and Hydra cluster $(\ell, v) \approx\left(270^{\circ}, 3800 \mathrm{~km} \mathrm{~s}^{-1}\right)$ above the GP (upper left panel) with the Pavo II cluster $(\ell, v) \approx$ $\left(332^{\circ}, 4200 \mathrm{~km} \mathrm{~s}^{-1}\right)$ below the GP (lower left panel). This entire large-scale structure is the optical counterpart of the Great Attractor. The Centaurus-Crux cluster (almost dead centre in the middle left panel) at $(\ell, v) \approx\left(306^{\circ}, 6200 \mathrm{~km} \mathrm{~s}^{-1}\right)$ is also part of the GA. The MEFOS observations of the Norma cluster form the basis for a detailed dynamical study of this rich and nearby cluster at the heart of the Great Attractor. Recently, 
we have obtained $2 \mathrm{dF}$ spectroscopy of the Norma cluster (primarily for velocity dispersion measurements of the elliptical galaxies in the cluster in order to determine the distance to the Norma cluster via the Fundamental Plane analysis). The results of this - and the dynamical analysis of the cluster - will be presented in a separate paper.

Out to $30000 \mathrm{~km} \mathrm{~s}^{-1}$, many clusters in the velocity range $12500-20000 \mathrm{~km} \mathrm{~s}^{-1}$ are visible (middle right panel), the most prominent is the Ara cluster at $(\ell, v)=\left(329^{\circ}, 14722 \mathrm{~km} \mathrm{~s}^{-1}\right)$.

\section{Summary}

Multi-fibre spectroscopy with MEFOS on the 3.6-m telescope of ESO has resulted in 764 redshifts in the southern Zone of Avoidance. Contrary to the SAAO and Parkes components of our redshift survey in the Zone of Avoidance, MEFOS has focussed on the faint end of the galaxies in our optical galaxy catalogue in the high galaxy-density regions. As a result, MEFOS has contributed significantly to the knowledge of low Galactic latitude clusters at $v \gtrsim 12500 \mathrm{~km} \mathrm{~s}^{-1}$.

Acknowledgements. This research has made use of the NASA/IPAC Extragalactic Database (NED), which is operated by the Jet Propulsion Laboratory, Caltech, under contract with the National Aeronautics and Space Administration. PAW kindly acknowledges financial support from the Antares fund and from the National Research Foundation. RCKK thanks CONACyT for their support (research grant 27602E). We thank the Referee, Dr. R. Peletier, for the many useful comments and suggestions. Part of this survey was performed at the Kapteyn Astronomical Institute of the University of Groningen and at the Observatoire de Paris-Meudon. Their support is greatfully acknowledged.

\section{References}

Abell, G. O., Corwin, H. G., \& Olowin, R. P. 1989, ApJS, 70, 1

Batuski, D. J., Miller, C. J., Slinglend, K. A., et al. 1999, ApJ, 520, 491

Colless, M., Dalton, G., Maddox, S., et al. 2001, MNRAS, 328, 1039

Cuby, J. G., \& Mignoli, M. 1994, Proc. SPIE, 2198, 98

Dressler, A., Faber, S. M., Burstein, D., et al. 1987, ApJ, 313, 37

Ebeling, H., Mullis, C. R., \& Tully, R. B. 2002, ApJ, 580, 774
Fairall, A. P., Woudt, P. A., \& Kraan-Korteweg, R. C. 1998, A\&AS, 127, 463 (Paper II)

Felenbok, P., Guérin, J., Fernandez, A., et al. 1997, Exp. Astron., 7, 65

Hasegawa, T., Wakamatsu, K., Malkan, M., et al. 2000, MNRAS, 316, 326

Hill, J. M., \& Oegerle, W. R. 1993, AJ, 106, 831

Juraszek, S. J., Staveley-Smith, L., Kraan-Korteweg, R. C., et al. 2000, AJ, 119, 1627

Kolatt, T., Dekel, A., \& Lahav, O. 1995, MNRAS, 275, 797

Kraan-Korteweg, R. C. 2000, A\&AS, 141, 123

Kraan-Korteweg, R. C., \& Lahav, O. 2000, A\&ARv, 10, 211

Kraan-Korteweg, R. C., \& Woudt, P. A. 1994, in Unveiling LargeScale Structures behind the Milky Way, ed. C. Balkowski \& R. C. Kraan-Korteweg, ASP Conf. Ser., 67, 89

Kraan-Korteweg, R. C., Cayatte, V., Balkowski, C., et al. 1994, in Unveiling Large-Scale Structures Behind the Milky Way, ed. C. Balkowski \& R. C. Kraan-Korteweg, ASP Conf. Ser., 67, 99

Kraan-Korteweg, R. C., Fairall, A. P., \& Balkowski, C. 1995, A\&A, 297, 617 (Paper I)

Kraan-Korteweg, R. C., Henning, P. A., \& Schröder, A. 2002, A\&A, 391,887

Kraan-Korteweg, R. C., Woudt, P. A., Cayatte, V., et al. 1996, Nature, 379,519

Lauberts, A. 1982, The ESO/Uppsala Survey of the ESO (B) Atlas (Garching: ESO)

Lynden-Bell, D., Faber, S. M., Burstein, D., et al. 1988, ApJ, 326, 19

McHardy, I. M., Lawrence, A., Pye, J. P., et al. 1981, MNRAS, 197, 893

Pinkney, J., Rhee, G., Burns, J. O., et al. 1993, ApJ, 416, 36

Saunders, W., D'Mellow, K. J., Valentine, H., et al. 2000, in Mapping the Hidden Universe: the Universe behind the Milky Way - the Universe in HI, ed. R.C. Kraan-Korteweg, P. A. Henning \& H. Andernach, ASP Conf. Ser., 218, 141

Schlegel, D. J., Finkbeiner, D. P., \& Davis, M. 1998, ApJ, 500, 525

Tonry, J. L., \& Davies, R. L. 1979, AJ, 84, 1511

Tonry, J. L., Blakeslee, J. P., Ajhar, E. A., et al. 2000, ApJ, 530, 625

de Vaucouleurs G., de Vaucouleurs, A., \& Corwin, H. G. 1976, Second Reference Catalogue of Bright Galaxies, Univ. Texas Monographs in Astron. No. 2 (Austin: Univ. of Texas)

Visvanathan, N., \& Yamada, T. 1996, ApJS, 107, 521

Woudt, P. A. 1998, Ph.D. Thesis, University of Cape Town

Woudt, P. A., \& Kraan-Korteweg, R. C. 2001, A\&A, 380, 441

Woudt, P. A., Kraan-Korteweg, R. C., \& Fairall, A. P. 1999, A\&A, 352, 39 (Paper III)

Zehavi, I., Blanton, M. R., Frieman, J. A., et al. 2002, ApJ, 571, 172 